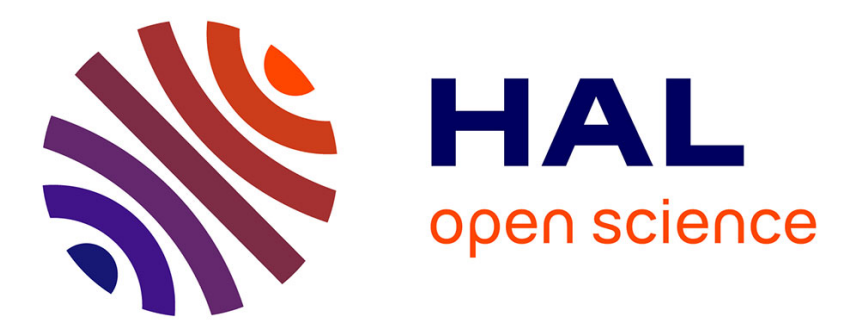

\title{
Limitations of simple flow models for the simulation of nanoimprint
}

\author{
Hubert Teyssedre, Pierre Gilormini, Gilles Regnier
}

\section{To cite this version:}

Hubert Teyssedre, Pierre Gilormini, Gilles Regnier. Limitations of simple flow models for the simulation of nanoimprint. International Polymer Processing, 2013, 28, pp.72-78. 10.3139/217.2673 . hal-00799731

\section{HAL Id: hal-00799731 \\ https://hal.science/hal-00799731}

Submitted on 13 Mar 2013

HAL is a multi-disciplinary open access archive for the deposit and dissemination of scientific research documents, whether they are published or not. The documents may come from teaching and research institutions in France or abroad, or from public or private research centers.
L'archive ouverte pluridisciplinaire HAL, est destinée au dépôt et à la diffusion de documents scientifiques de niveau recherche, publiés ou non, émanant des établissements d'enseignement et de recherche français ou étrangers, des laboratoires publics ou privés. 


\title{
Limitations of Simple Flow Models for the Simulation of Nanoimprint
}

\author{
H. Teyssèdre ${ }^{1,2}$, P. Gilormini*1 ${ }^{*}$ and G. Régnier ${ }^{1}$ \\ ${ }^{1}$ PIMM, Arts et Métiers ParisTech, CNRS, Paris, France \\ ${ }^{2}$ Silsef, Archamps, and CEA/LETI, Grenoble, France
}

\begin{abstract}
A quick evaluation of the forces involved in nanoimprint would be very helpful in the prevention of mold deflection. Unfortunately, it is shown here that assuming simplified flows may lead to quite incorrect evaluations of these forces, even for simple periodic patterns and a Newtonian behavior. The mere use of the classical result of the lubrication theory does not account for the range of thickness-to-width ratios that may be involved, especially at the beginning of the process. An improved squeeze model includes this effect, but still underestimates the imprint force. Moreover, finite element simulations demonstrate limitations of two more elaborate models that are found in the literature. These simulations also show that two flow modes can be defined, according to whether or not the polymer touches the mold sidewalls. A deeper analysis of these two modes may help the definition of a more appropriate simplified model in the future.
\end{abstract}

Keywords nanoimprint; finite elements; simplified models.

\section{Introduction}

Nanoimprint lithography is a process introduced by Chou et al. (1995), where nanometric patterns are engraved into a very thin polymer film. In the variant considered here, thermal nanoimprint, the polymer is spin coated on a substrate before impression at high temperature. The surfaces involved are of the order of hundreds of square centimeters to allow for the simultaneous

*Mail address: Pierre Gilormini, PIMM, Arts et Métiers ParisTech, CNRS, 151 bvd de l'Hôpital, 75013 Paris, France (pierre.gilormini@ensam.eu).

Published in: International Polymer Processing, vol. 28, pp. 72-78 (2013). 
impression of a very large number of patterns, and defects may arise from mold deflection over relatively long distances. This motivates the study of the force that the flowing polymer applies on a single protrusion of a rigid periodic mold, in a first step. In further developments, a quick evaluation of this force may be useful to study mold deflection at length scales larger than the pattern period, with each protrusion replaced by a time-varying point force, for instance.

Two approaches are possible to evaluate this force. The first one relies on analytical expressions which allow putting more computational resource at the upper level of mold deflection. The risk is a too crude evaluation of the imprint force involved in the many configurations that may arise. Basically, results taken from the lubrication theory are employed in this context. For instance, Schultz et al. (2006) adapted this theory to model the flow below an elementary periodic pattern, and Leveder et al. (2007) used it in a reverse manner to deduce the polymer viscosity from the applied force. The other approach, which allows especially to overcome the limitation of the lubrication theory to small thickness-to-width aspect ratios below patterns, performs a full numerical simulation of the polymer flow like in Jeong et al. (2002), or Rowland et al. (2005), for example. These authors put emphasis on polymer flow but did not provide the corresponding imprint force, though, whereas Young (2005) did compute it. Of course, this type of approach gives a precise solution to a given flow problem, provided the discretization is fine enough, but it requires the availability of a simulation code and involves much longer computation times than using analytical estimates. It may also be very useful to validate the latter.

Nanoimprint is a complex process that involves not only fluid-solid coupling, as already mentioned above, but also surface tension phenomena and nonlinear fluid behavior, among other complexities. This paper considers an idealized problem where surface tension is neglected and a constant viscosity can be assumed, and emphasis is put on some simple approximate solutions 
to that problem, using finite elements as a reference solution. Comparison with experimental results would allow to discuss the pertinence of the idealized problem considered but is beyond the present work, which is limited to simulation. The aim is to guide the development of simple solutions before more complexity, like a nonlinear behavior for instance, can be included in a further stage.

This paper addresses the applicability of very simple velocity fields to evaluate the imprint force when the initial polymer thickness is not necessarily much smaller than the protrusion width. First, nanoimpression is modeled as a squeeze flow and a velocity field is proposed that extends to thick films the well-known velocity field that is used in the lubrication theory for thin films. Then, finite element simulations are performed. They allow to discuss the validity of the analytical expression obtained and the relevance of squeeze flows to model nanoimpression. In addition, they demonstrate that two models of the literature that use simple flows are not satisfactory for substantial initial polymer thicknesses. This leaves the problem of a simple evaluation of the imprint force open, except for very limited geometries.

\section{Problem Statement}

Nanoimprint lithography can be used to reproduce a variety of patterns, like periodic arrays of pillars or holes with circular or square cross-sections, among others (see Guo, 2004, for examples), but simple lines are very frequently used to demonstrate the technique, as in the pioneering papers by Chou et al. (1995, 1996), and to test models (Jeong et al., 2002; Rowland et al., 2005; Young, 2005, for instance). One advantage of line patterns is the two-dimensional flow involved, which allows the study of a mere cross section. Moreover, the study can be limited to a single period when the pattern is periodic, and even to half a period for symmetry reasons, as shown in Fig. 1c and 1d. Fig. 1a illustrates also another advantage of simple line patterns, which is a limited number of geometric parameters. The pattern 
(a)
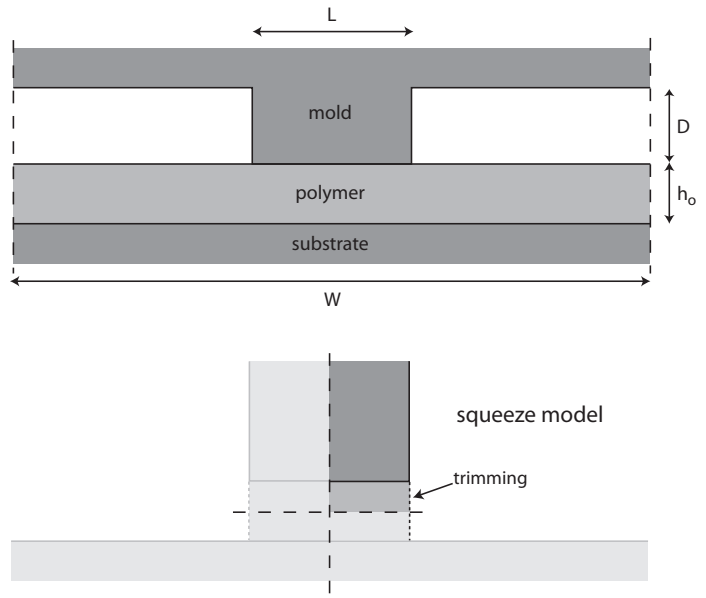

(c)



(d)

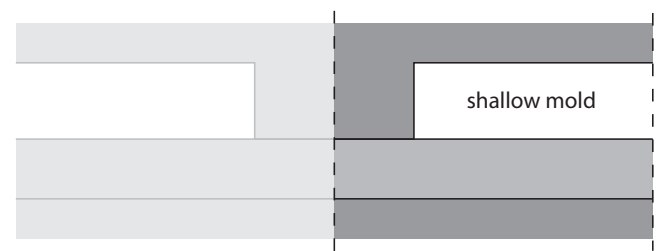

Figure 1: Before the mold is moved down, cross section of a single period for the nanoimpression of a simple line pattern (a) and of the minimal parts that need being considered (darker gray shades) for the squeeze model (b), and for deep (c) or shallow (d) patterns. Dashed lines define symmetry axes.

is characterized by its pitch $(W)$, width $(L)$ and depth $(D)$, and the initial thickness of the polymer $\left(h_{0}\right)$ defines the last geometric parameter of the problem.

The polymer is defined as a linear viscous fluid in this study, and its viscosity $\eta$ is the only material parameter, as in Leveder at al. (2007) or Jeong et al. (2002), although power-law viscous fluids were considered in the finite element simulations of Rowland et al. (2005) and in the analytical approach of Hsin and Young (2008), among others. Actually, as shown 
by Schultz et al. (2006), for instance, the polymer exhibits shear-thinning above a transition shear rate, but the limitation to a linear behavior may be acceptable for low strain rates. Moreover, the purpose of the present study is to explore the applicability of simple models, and this can be limited to simple behaviors in a first stage. For the same reason, and like in many other studies, surface tension is neglected here, although it may play an important role at the nano scale and has been taken into account by Jeong et al. (2002) and Rowland et al. (2005), among others. A consequence of these assumptions is that the process parameter can be indifferently either the downward velocity $V$ of the mold or the force $F$ applied per unit pattern length, since these two quantities are proportional when the fluid is Newtonian. We will prefer a constant $V$ as process parameter, for its convenience when finite elements are employed.

Finally, inertial effects and gravity can be neglected in microflows and nanoflows of viscous liquids (Karniadakis et al., 2005, for example), where extremely low Reynolds numbers are involved, and a Stokes flow without body forces is considered here, consequently.

\section{Squeeze Model}

An approximate model for the two-dimensional flow between mold and substrate can be defined by the squeeze process defined in Fig. 1b, like in Leveder et al. (2007) for instance. This amounts to continuously trimming off, during the mold descent, the polymer volume that is not located beneath the protrusion. Therefore, the imprint force is likely to be underestimated, the mold depth $D$ has no effect, and the force will depend on the current thickness $h$ of the film, without any history effect related to its initial value $h_{0}$.

Using a coordinate system with its origin at the symmetry center of the section considered which, consequently, moves vertically with velocity $V / 2$, a velocity field can be adapted from the axisymmetric case treated by 
Engmann et al. (2005) as

$$
v_{x}=V\left(\frac{3 x}{2 h}-\frac{6 x y^{2}}{h^{3}}\right) \quad \text { and } \quad v_{y}=-V\left(\frac{3 y}{2 h}-\frac{2 y^{3}}{h^{3}}\right)
$$

which does give $v_{x}(y=-h / 2)=v_{x}(y=h / 2)=0$ and $v_{y}(y=-h / 2)=$ $-v_{y}(y=h / 2)=V / 2$ for the no-slip boundary condition, and leads to the following non-zero strain rate components:

$$
\dot{\varepsilon}_{x x}=-\dot{\varepsilon}_{y y}=3 V\left(\frac{1}{2 h}-\frac{2 y^{2}}{h^{3}}\right) \quad \text { and } \quad \dot{\varepsilon}_{x y}=\dot{\varepsilon}_{y x}=-6 V \frac{x y}{h^{3}}
$$

where $\dot{\varepsilon}_{x x}=-\dot{\varepsilon}_{y y}$ expresses incompressibility. The nonzero in-plane components of the stress tensor

$$
\begin{gathered}
\sigma_{x x}=-\frac{\eta V}{2 h^{3}}\left(36 y^{2}-12 x^{2}-3 h^{2}+3 L^{2}\right) \\
\sigma_{y y}=\frac{\eta V}{2 h^{3}}\left(12 y^{2}+12 x^{2}-9 h^{2}-3 L^{2}\right) \\
\text { and } \sigma_{x y}=\sigma_{y x}=-12 \eta V \frac{x y}{h^{3}}
\end{gathered}
$$

which result from the combination $\sigma_{i j}=2 \eta \dot{\varepsilon}_{i j}-p \delta_{i j}$ of (2) and of the field of hydrostatic pressure

$$
p=\frac{3 \eta V}{2 h^{3}}\left(4 y^{2}-4 x^{2}+h^{2}+L^{2}\right)
$$

are such that the equations of fluid motion for plane Stokes flow, $\partial \sigma_{x x} / \partial x+$ $\partial \sigma_{x y} / \partial y=0$ and $\partial \sigma_{y y} / \partial y+\partial \sigma_{y x} / \partial x=0$, are checked. The boundary conditions $\sigma_{x x}=0$ and $\sigma_{x y}=0$ are not satisfied everywhere on the lateral free surfaces (at $x= \pm L / 2$ ), but they are satisfied on average, since (3) leads to

$$
\int_{-h / 2}^{h / 2} \sigma_{x x}( \pm L / 2, y) \mathrm{d} y=0 \quad \text { and } \quad \int_{-h / 2}^{h / 2} \sigma_{x y}( \pm L / 2, y) \mathrm{d} y=0 .
$$

These are the only approximations involved in the solution proposed here to the squeeze problem considered. The force that is applied to the substrate (opposite to the force applied to the mold) per unit length (normal to the $(x, y)$ section considered) yields from (3):

$$
F=\int_{-L / 2}^{L / 2} \sigma_{y y}(x, h / 2) \mathrm{d} x=\eta V\left(\frac{L^{3}}{h^{3}}+3 \frac{L}{h}\right) .
$$


When $h \ll L$, the classical result of the lubrication theory used by Leveder et al. (2007), for example, is recovered:

$$
F_{L}=\eta V\left(\frac{L}{h}\right)^{3}
$$

Taking this expression as a reference, the following normalized expression of (6) will be employed:

$$
\frac{F}{F_{L}}=1+3\left(\frac{h}{L}\right)^{2}
$$

The Abaqus (2009) finite element code offers the possibility, among numerous features, to simulate the flow of quasi-incompressible viscous fluids, including the Newtonian special case. In the volume of fluid method used, two parameters must be prescribed that are not listed above: a density and a velocity for wave propagation, which are of crucial importance for the time step used. The latter is excessively small if realistic values are taken for the density and wave speed, because of the length scale involved in nanoimpression. Therefore, dummy values were defined, which did not alter significantly the initial problem to solve and allow reasonable computation times. First, the density was chosen in order to have a Reynolds number of 0.1 , since this was checked to be a good approximation to a Stokes flow (zero Reynolds number) in our case. Then, the wave speed was adjusted to get both a good volume preservation and time increments that lead to reasonable computation times. The necessarily three-dimensional mesh was defined as one layer of a regular array of identical small cubes, each one being an Eulerian element with 8 nodes and reduced integration. Two-dimensional flows were obtained by prescribing suitable symmetry conditions on the front and rear faces of the one-element thick mesh. The trimming process involved in the squeeze model was obtained trivially by letting the fluid cross the lateral sides of the mesh. The mold was defined as a rigid body with a prescribed downward velocity and no-slip boundary condition.

The results of the finite element simulations are shown in Fig. 2. As expected, the lubrication theory, which has been developed for thin films, 


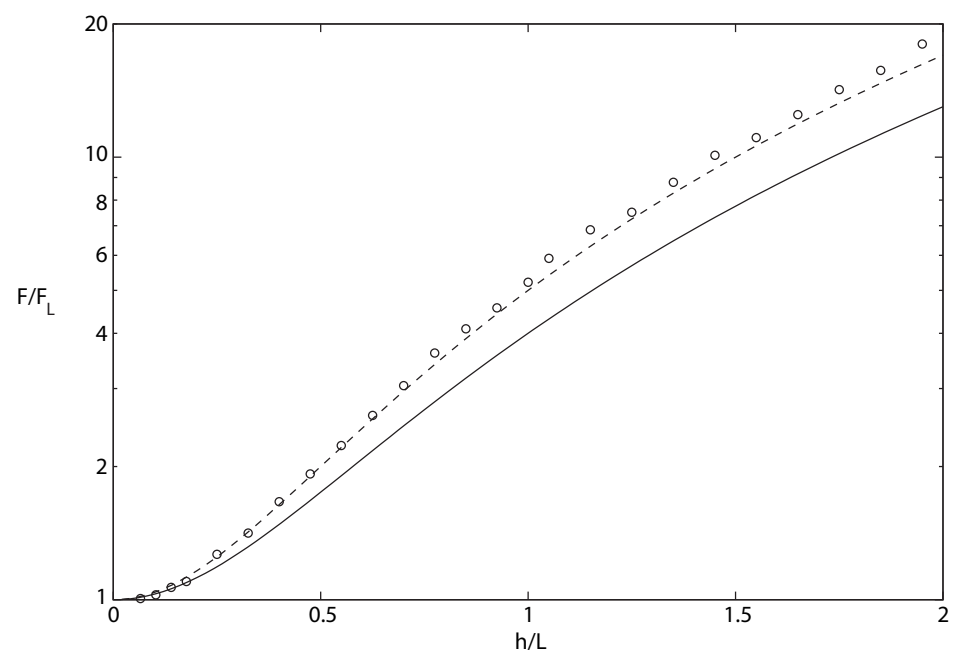

Figure 2: Applied force normalized by the lubrication theory result for twodimensional squeeze with thickness-to-width aspect ratio $h / L$. Comparison between finite element simulations (symbols) and the uncorrected (eqn. (8), solid line) or corrected (eqn. (9), dashed line) analytical squeeze model.

gives a very bad estimate of the pressing force for thick films, leading to strongly underestimated values. For instance, a difference by a factor of 5 is obtained for $h / L=1$. This clearly shows that the results of the lubrication theory are not sufficient for simulating the early stages of nanoimpression, when $h / L$ is not yet small, and would lead for example to an overestimated mold velocity for a fixed applied force. Fig. 2 also shows that the above approximate model for thick films (8) improves very significantly over the lubrication theory, but still underestimates the force.

In addition to the $h / L \ll 1$ case, another simple situation can be considered where an analytical solution is asymptotically exact, which is the $h / L \gg 1$ case. For such a geometry, the effect of the fluid-solid contact conditions tends to localize near the top and bottom of the slender fluid column, with a negligible influence on the overall force. Therefore, the solution with a uniform strain rate that pertains to perfect slip (zero shear stress on both the protrusion and the substrate) should provide a good estimate, which gives $F=4 \eta V L / h$ trivially. This suggests a very simple empirical 
modification of (8) as

$$
\frac{F}{F_{L}}=1+4\left(\frac{h}{L}\right)^{2}
$$

which is shown in Fig. 2 to lead actually to a very good agreement with the finite element results in the $h / L$ range considered.

\section{Finite Element Simulation of Nanoimprint}

The trimming process involved in the squeeze model described in the previous section is likely to underestimate the imprint force, but to which extent? Finite element simulations have been conducted to answer this question, by considering a deep mold as shown in Fig. 1c. This introduces the pattern pitch $W$ as a new parameter. Initially, the amount of polymer that opposes the lateral flow occurring below the protrusion will depend on $W$ and on the initial polymer thickness $h_{0}$. Later in the impression process, for a polymer thickness $h<h_{0}$ below the protrusion, volume preservation implies that a fraction of the polymer is located above the protrusion level, and this may increase the force further. In these conditions, the current force may not be a function of $h$ only, but also of $h_{0}$. This dependence was not present in the squeeze model.

Fortunately, the finite element simulations that we performed demonstrate that the influence of $h_{0}$ can be neglected below a value that depends on the $W / L$ ratio. For a ratio of $W / L=2$, for instance, Fig. 3 shows that the evolution of the applied force is a function of $h / L$ only provided that $h_{0} / L$ is below a value of about 0.6 , since the forces obtained are close to a single curve (open symbols in Fig. 3). This means that the polymer located above the protrusion level does not contribute significantly to the force required for the imprint process to carry on. We will call this situation a "mode A" flow, for which a simplified model may asssume that Fig. 1c applies for any $h / L$ below the critical $h_{0} / L$ value. Unfortunately, the modified squeeze model (9) of the previous section is too crude for this purpose and largely 


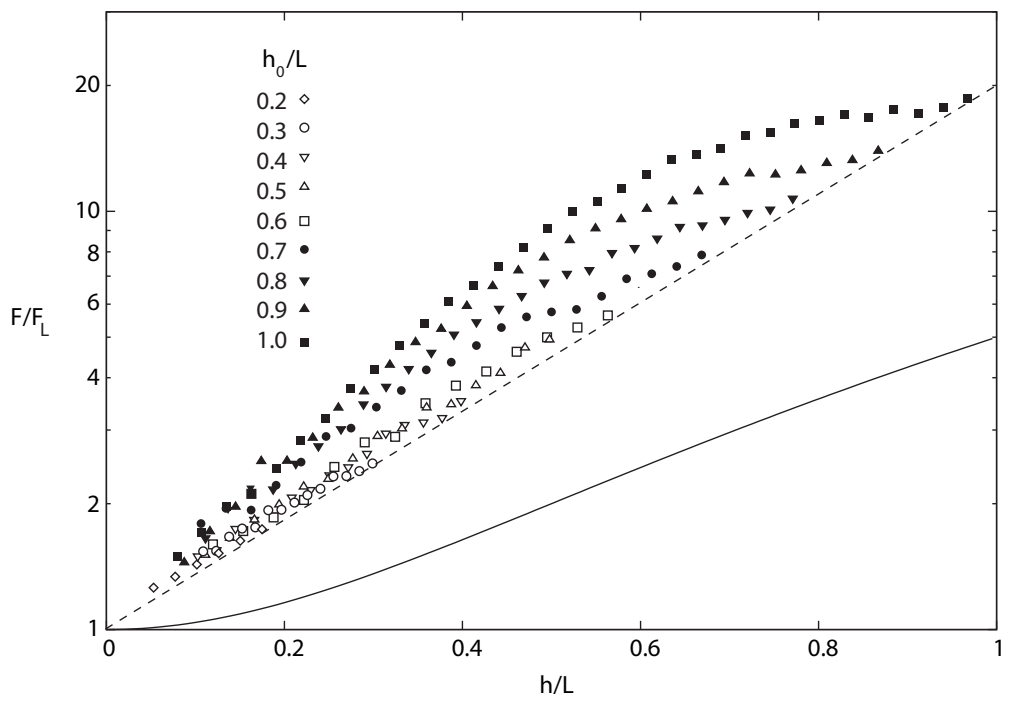

Figure 3: Applied force computed by finite elements and normalized by the lubrication theory result for nanoimpression using a deep pattern with a $W / L$ ratio of 2 and starting from various $h_{0} / L$ values. Comparison with the corrected analytical squeeze model (eqn. (9), solid line). The straight dashed line is just a guide for the eye.

underestimates the nanoimpression force, as can be seen in Fig. 3.

For an initial thickness beyond the critical value (solid symbols in Fig. 3), the nanoimpression force still starts from an extension of the result for mode A, which corresponds to such configurations as in Fig. 1c, but then the force keeps above the curve obtained for mode A and is higher for larger initial thicknesses, as can be observed in Fig. 3. This corresponds to a change in the flow mode. Below the critical $h_{0} / L$ value (open symbols in Fig. 3 ), in mode A, the velocity of the polymer at the protrusion corner is inclined but not vertical, and the flow does not contact the mold sidewalls, leading to such profiles as in Fig. 4a. In contrast, the velocity is found vertical and contact occurs for larger $h_{0} / L$ values (solid symbols in Fig. 3), which defines a mode $\mathrm{B}$ and leads to Fig. 4b, for instance. The shear induced by this additional contact increases the imprint force. Moreover, volume preservation implies, for a given $h / L$ value, a longer contact length if the initial thickness is larger, which leads to a higher imprint force. 


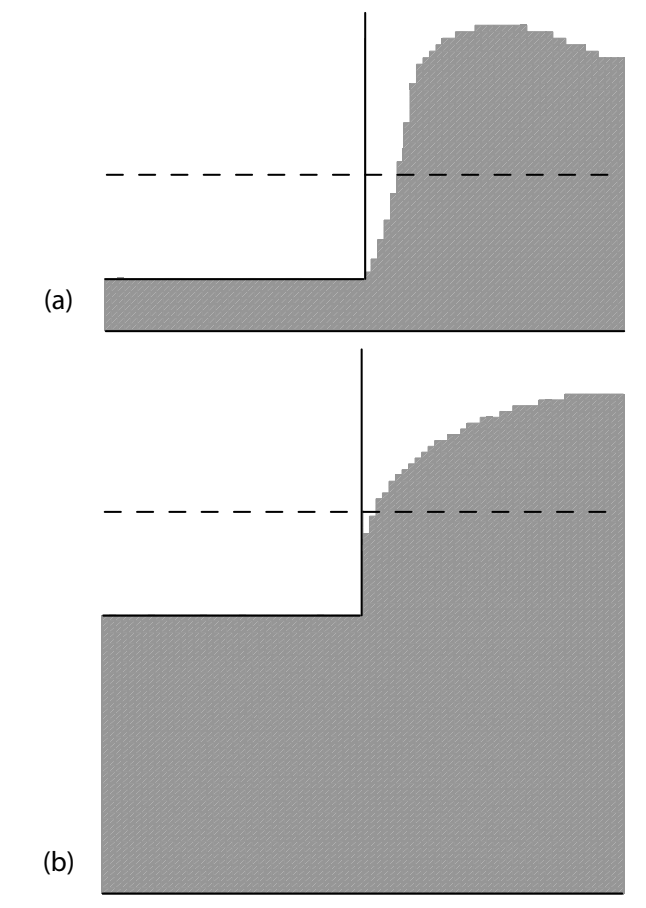

Figure 4: Deformed configurations computed with the finite element method for a deep mold (solid line) with $W / L=2$ and either $h_{0} / L=0.3$ (a) or $h_{0} / L=0.8$ (b), for the same mold displacement. These are examples of flow modes A and B, respectively. Dashed line: initial surface of the polymer.

When $h / L$ becomes extremely small, the force required to squeeze the very thin film under the protrusion increases without limit, since it is proportional to $(L / h)^{3}$ as mentioned in section 3 , and the additional force induced by the polymer located beyond the protrusion, with or without contact with its sidewalls, becomes negligible comparatively. This justifies the plots to end at the origin in Fig. 3.

A straight line has been obtained for mode A when $W / L=2$ with the axes used in Fig. 3, at least in the $h / L$ range considered, but this was incidental, since Fig. 5 shows a curved line when $W / L=4$, i.e. for a larger distance between successive protrusions. The other trends are qualitatively similar to Fig. 3, with the modified squeeze model still underestimating the force strongly but with the transition between the two flow modes appearing 


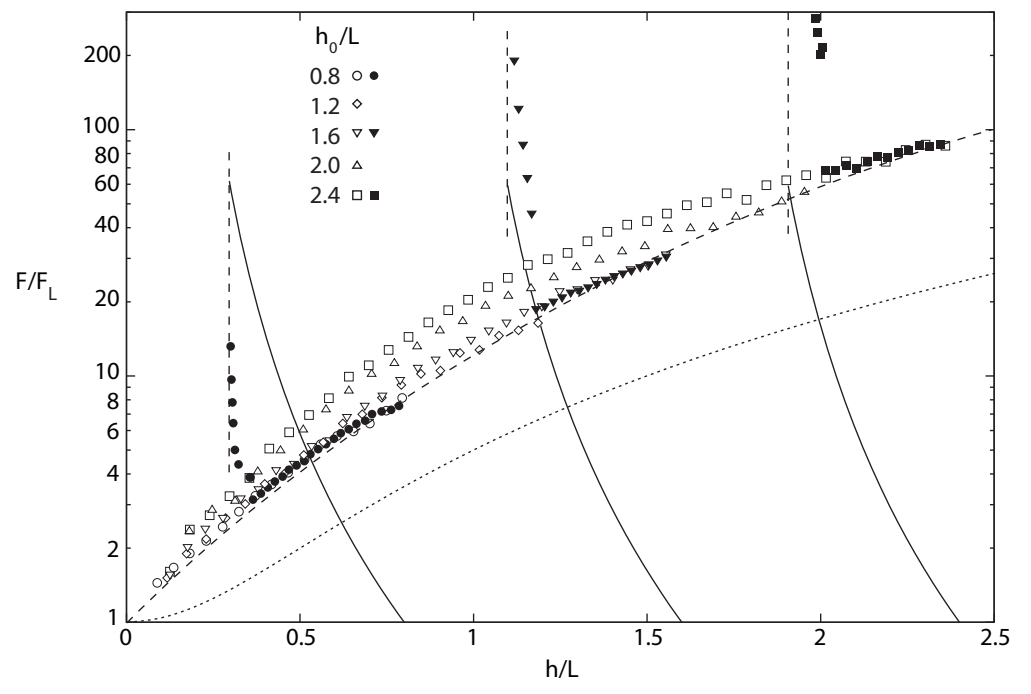

Figure 5: Applied force computed by finite elements and normalized by the lubrication theory result for nanoimpression with a $W / L$ ratio of 4 , starting from various $h_{0} / L$ values and using either a deep mold (open symbols) or a shallow mold with $D / L=2 / 3$ (solid symbols). Comparison with the corrected analytical squeeze model for deep mold (eqn. (9), dotted line) and with the model of Schultz et al. (2006) for shallow mold (solid lines). The vertical dashed lines define the $h$ values for complete cavity filling. The curved dashed line is just a guide for the eye.

for a larger $h_{0} / L$ value, about 1.8. Simulations were also carried out, for $W / L=4$, with a shallow pattern (see Fig. 1d) having a depth of $D / L=2 / 3$. This corresponds to a filled cavity for a mold descent of $L / 2$, since volume preservation leads to a complete filling for

$$
h=h_{0}-D\left(1-\frac{L}{W}\right) .
$$

Fig. 5 compares the evolutions of the force using a shallow pattern or using a deep pattern (solid vs. open symbols), for three different initial thicknesses $h_{0} / L$. Of course, the evolutions are identical before the polymer touches the top of the cavity. Then, the force increases sharply for a shallow pattern, whereas it keeps decreasing for a deep pattern with the normalization used. The force increase after the polymer free surface has touched the top of the cavity reduces even more the applicability of the lubrication theory, since 


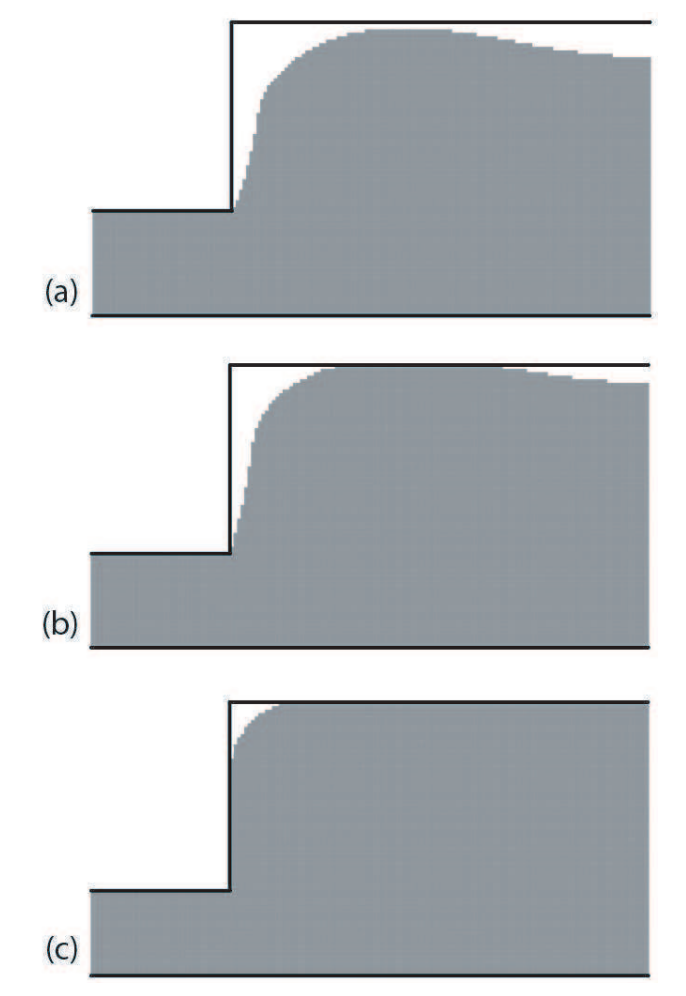

Figure 6: Cavity filling obtained for $h_{0} / L=0.8, W / L=4$ and $D / L=2 / 3$. Mold descent of $0.41 \mathrm{~L}$ (a), $0.45 \mathrm{~L}$ (b) and $0.49 \mathrm{~L}$ (c). Complete filling is obtained for a descent of $L / 2$.

$F / F_{L}$ keeps far from 1 all along the process, except possibly for very thin initial polymer layers for which the cavity would be filled after extremely small polymer thicknesses are obtained.

The kinematics of the end of cavity filling is illustrated in Fig. 6 for a mode A flow $\left(h_{0} / L=0.8\right)$. First, the top of the free surface is modified, with both its part close to the symmetry axis and its lowest part almost unaffected. Then, the lowest part becomes steeper and closer to the sidewall. Finally, the only left space is in the corner of the cavity, with the polymer shearing along the sidewall, and this is similar to the configurations obtained directly with mode $\mathrm{B}$ flows (for large $h_{0} / L$ values, where the peak of the free surface is along the symmetry axis). 


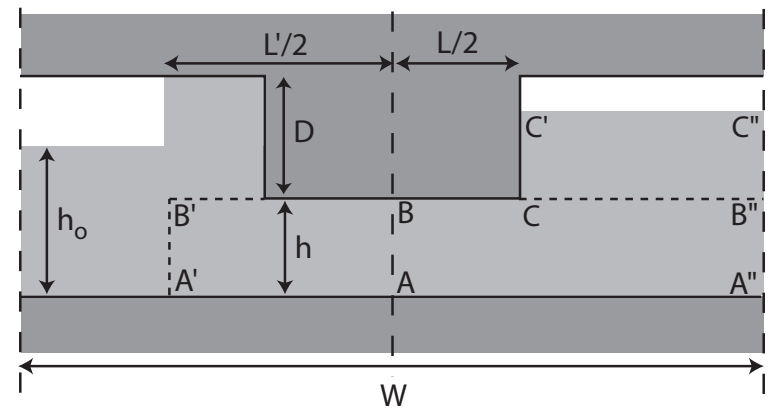

Figure 7: Notations used to define the models of Schultz et al. (2006), on the left, and of Hsin and Young (2008), on the right.

\section{Discussion}

The above definition of two flow modes when imprinting with a deep periodic mold can be compared with the results obtained by Rowland et al. (2005). These authors also defined two flow modes, but with the free surface within one pattern period showing either one or two peaks. Fig. 4a, for instance, is an example of a two-peak mode, whereas Fig. 4b illustrates a one-peak mode (the highest polymer level is obtained on a symmetry axis). This definition puts more emphasis on the free surface shape than on the imprint force, and Rowland et al. (2005) found that the mode change is obtained for $h_{0} / L=(W / L-1) / 2.4$ with our notations. This gives $h_{0} / L \approx 0.4$ when $W / L=2$, and we did obtain a dual-peak surface for $h_{0} / L=0.2$ and 0.3 , and a single peak for $h_{0} / L=0.4$ and beyond, but contact with the protrusion sidewalls nevertheless occurred for $h_{0} / L=0.7$ at least. This indicates that mode transition for the force occurs for $h_{0} / L$ values that are larger than for the shape transition observed by Rowland et al. (2005), since single-peak free surfaces may be associated with mode A flow. This is confirmed in the $W / L=4$ case of Fig. 5, where Rowland et al. (2005) would predict a change in peak numbers for $h_{0} / L=1.25$, while we obtained a transition for $h_{0} / L \approx 1.8$ for the force evolution.

A first model that uses a simplified flow and which can be compared 
with the finite element results is that of Schultz et al. (2006). As indicated in Fig. 7, it assumes that the volume of polymer that is occupied by the new position of the mold is moved near the corner of the mold. Accordingly, this model requires a shallow mold and implies that the initial polymer free surface is maintained beyond the redistributed volume, as shown in Fig. 7. Volume preservation provides the polymer-mold contact width $L^{\prime}$ readily, and the model considers a squeeze of the polymer in the $\mathrm{ABB}^{\prime} \mathrm{A}^{\prime}$ region, using the lubrication theory result, which leads to the total force per unit length

$$
F_{S}=\eta V\left(\frac{L^{\prime}}{h}\right)^{3}=\eta V\left(\frac{L}{h}\right)^{3}\left(\frac{D}{D-h_{0}+h}\right)^{3} .
$$

For deep molds $(D / L \gg 1)$, this model recovers the elementary squeeze model (7) that was shown in section 3 to underestimate the force. When normalized by $F_{L}$, the force on shallow molds is equal to 1 when $h=h_{0}$, i.e. the force evolution starts from the lower axis in Fig. 5, which is far too low compared to the finite element results, and it equals $(W / L)^{3}$ for any $h_{0}$ value when the cavity is filled, according to (10). Moreover, the $F_{S} / F_{L}$ ratio keeps increasing when $h$ decreases, whereas Fig. 5 (solid symbols) shows a decrease before the ratio increases up to complete cavity filling. It may thus be concluded from the comparison performed in Fig. 5 in three cases that the very simple flow assumed by Schultz et al. (2006) is not realistic enough and does not allow a good evaluation of the nanoimpression force involved with shallow molds for the $h_{0} / L$ ratios considered here, but it must be mentioned that the simulations presented by Schultz et al. (2006) are limited to $h_{0} / L \leq 0.25$.

A different simplified polymer flow is considered by Hsin and Young (2008) for power-law fluids, where the free surface of the polymer is assumed to keep parallel to the substrate, as shown in Fig. 7, and where an example with $h_{0} / L=1.05$ is given. In the simple case of a Newtonian behavior, horizontal and vertical plane Poiseuille flows are assumed in the $\mathrm{AA}^{\prime \prime} \mathrm{B}^{\prime \prime} \mathrm{B}$ region and in twice the $\mathrm{CB}^{\prime \prime} \mathrm{C}^{\prime \prime} \mathrm{C}^{\prime}$ region (since $\mathrm{B}^{\prime \prime} \mathrm{C}^{\prime \prime}$ is a symmetry axis), 
respectively, leading to pressure drops

$$
p_{0}-p_{1}=12 \eta \frac{Q}{h^{3}} \frac{W}{2} \quad \text { and } \quad p_{2}=12 \eta \frac{Q^{\prime}}{(W-L)^{3}} W \frac{h_{0}-h}{W-L}
$$

where $p_{0}, p_{1}$ and $p_{2}$ denote pressure along $\mathrm{AB}, \mathrm{A}^{\prime \prime} \mathrm{B}^{\prime \prime}$ and $\mathrm{B}^{\prime \prime} \mathrm{C}$, respectively. The above computation of $p_{2}$ assumed a zero pressure on the free surface $\mathrm{C}^{\prime} \mathrm{C}^{\prime \prime}$ and applied the preservation of volume to compute the $\mathrm{CC}^{\prime}$ length. In these expressions, $Q$ and $Q^{\prime}$ denote planar flow rates (per unit mold length normal to the cross section considered). Assuming now that $p_{2}=p_{1}$ and $Q^{\prime}=2 Q=V L$ (which leads for $p_{1} / p_{0}$ to the same expression as (6) of Hsin and Young, 2008, with different notations) gives

$$
p_{0}=3 \eta V W L\left[\frac{1}{h^{3}}+4 \frac{h_{0}-h}{(W-L)^{4}}\right] \quad \text { and } \quad p_{1}=12 \eta V W L \frac{h_{0}-h}{(W-L)^{4}} .
$$

Pressure $p_{1}$ applied along $\mathrm{CB}^{\prime \prime}$ equilibrates the shear force induced by the vertical plane Poiseuille flow on sidewall $\mathrm{CC}^{\prime}$ and induces a vertical force $F_{v}$. In addition, Hsin and Young (2008) compute the vertical force $F_{h}$ on BC due to the horizontal plane Poiseuille flow by applying $p_{0}$ uniformly (although pressure decreases linearly, actually). These two contributions lead to the following expression for the total force per unit length, for one period of the mold pattern:

$$
F_{H}=F_{h}+F_{v}=3 \eta V W L^{2}\left[\frac{1}{h^{3}}+4 \frac{W}{L} \frac{h_{0}-h}{(W-L)^{4}}\right]
$$

which does not tend to $F_{L}$ when $h \rightarrow 0$, but rather to $3 F_{L} W / L$. A comparison is made in Fig. 8 with finite element results in four cases where sidewall contact was established, and a strong disagreement appears clearly. It may then be concluded that the model of Hsin and Young (2008) is not appropriate to predict the imprint force.

The latter model, with its contributions from two distinct flows, can nevertheless be modified as follows. It may be noted first that assuming a plane Poiseuille flow in the $\mathrm{AA}^{\prime \prime} \mathrm{B}^{\prime \prime} \mathrm{B}$ region is not compatible with the symmetry conditions that apply along $\mathrm{AB}$ and $\mathrm{A}^{\prime \prime} \mathrm{B}^{\prime \prime}$. This suggests that the 




Figure 8: Applied force computed by finite elements and normalized by the lubrication theory result for nanoimpression using a deep pattern with a $W / L$ ratio of 2 and starting from various $h_{0} / L$ values (symbols). Comparison with the model of Hsin and Young (2008) (dashed lines) and with the partially predictive proposed model (solid lines).

horizontal flow considered in the model of Hsin and Young (2008), and the associated $F_{h}$ force, may contribute to the bad results obtained. Actually, the force due to the flow in the $\mathrm{AA}^{\prime \prime} \mathrm{B}^{\prime \prime} \mathrm{B}$ region corresponds to the $\mathrm{A}$ mode flow described in the previous section, and therefore it can be evaluated from the dashed lines shown in Figs. 3 and 5. Since a straight line was obtained for a $W / L$ ratio of 2 , the following fit yields readily

$$
F_{h}=10^{1.3 h / L} F_{L}
$$

which provides $F_{h}$ for this special $W / L$ value only. In contrast with the horizontal flow, the vertical flow assumed by Hsin and Young (2008) is in reasonable agreement with the velocity profiles found in the finite element simulations. This part of the Hsin and Young (2008) model should be corrected, though, because the flow rate $Q^{\prime}$ must be computed with respect to the sidewall, which is moving vertically with velocity $V$, for a relevant computation of the pressure drop. Therefore, $Q^{\prime}=V W$ applies, rather than 
$Q^{\prime}=V L$, as can be obtained by considering equivalently a fixed mold and a moving substrate with upward velocity $V$. Introducing this correction in (12) leads to the following vertical component for two sidewalls:

$$
F_{v}=12 \eta V W^{2} \frac{h_{0}-h}{(W-L)^{3}}
$$

with an amplifying factor of $W / L-1$ with respect to (14). The total force $F_{h}+F_{v}$ that results from (15) and (16) is shown in Fig. 8 as solid lines for four $h_{0}$ values. Although the agreement is still imperfect, which may be due to an interaction between the two flows that is not accounted by merely summing their contributions, one notes that the trends are correct, with the best accordance found among all models considered in this study. Unfortunately, this model is not fully predictive, since the contribution $F_{h}$ of mode A has to be obtained from finite element simulations. This should stimulate further effort to develop appropriate simple models for mode A, or to fit $F_{h}$ for a large set of $W / L$ ratios.

\section{Conclusion}

It has been shown that assuming simplified flows may lead to quite incorrect evaluations of the force involved in the nanoimprint of simple periodic patterns on Newtonian fluids for given mold velocity. It is recalled that an underestimation of the imprint force for a fixed mold velocity implies an overestimation of the mold velocity for a constant applied force, which is the usual experimental condition. The mere use of the classical result of the lubrication theory does not account for the range of thickness-to-width ratios that may be involved, especially at the beginning of the process. An improved squeeze model includes this effect, but still underestimates the imprint force. Moreover, finite element simulations have demonstrated limitations of two more elaborate models that are found in the literature.

These simulations have also shown that two flow modes could be defined, according to whether or not the polymer touches the mold sidewalls. 
A deeper analysis of these two modes may help the definition of a more appropriate simplified model in the future. Of course, additional work will still be required to account for shear-thinning and the effect of surface tension.

\section{Acknowledgements}

Support from the Agence Nationale de la Recherche through project SINCRONE is acknowledged.

\section{References}

Abaqus Software, version 6.9, Dassault Systèmes Simulia Corp., Providence RI, USA (2009)

Chou, S.Y., et al., "Imprint of Sub-25 nm Vias and Trenches in Polymers", Appl. Phys. Lett., 67, 3114-3116 (1995)

Chou, S.Y., et al., "Nanoimprint Lithography", J. Vac. Sci. Technol. B, 14, 4129-4133 (1996)

Engmann, J., et al., "Squeeze Flow Theory and Applications to Rheometry: A Review", J. Non-Newtonian Fluid Mech., 132, 1-27 (2005)

Guo, L.J., "Recent Progress in Nanoimprint Technology and its Applications", J. Phys. D: Appl. Phys., 37, R123-R141 (2004)

Hsin, I.-C., Young, W.-B., "Analysis of the Isothermal Compression in Nanoimprint Lithography Assuming a Power-Law Fluid", Int. Polym. Processing, 23, 24-29 (2008)

Jeong, J.-H., et al., "Flow Behavior at the Embossing Stage of Nanoimprint Lithography", Fibers Polym., 3, 113-119 (2002)

Karniadakis, G., et al.: Microflows and Nanoflows. Fundamentals and Simulations, Springer (2005)

Leveder, T., et al., "Flow Property Measurements for Nanoimprint Simulation”, Microelectronic Eng., 84, 928-931 (2007)

Rowland, H.D., et al., "Impact of Polymer Film Thickness and Cavity Size 
on Polymer Flow During Embossing: Toward Process Design Rules for Nanoimprint Lithography", J. Micromech. Microeng., 15, 2414-2425 (2005) Schulz, H., et al., "Impact of Molecular Weight of Polymers and Shear Rate Effects for Nanoimprint Lithography", Microelectronic Eng., 83, 259-280 (2006)

Young, W.-B., "Analysis of the Nanoimprint Lithography with a Viscous Model", Microelectronic Eng., 77, 405-411 (2005) 